\title{
Efficient, broadband and compact metal grating couplers for silicon-on-insulator waveguides
}

\author{
Stijn Scheerlinck, Jonathan Schrauwen, Frederik Van Laere, Dirk Taillaert, \\ Dries Van Thourhout and Roel Baets \\ Photonics Research Group, IMEC - Ghent University, Sint-Pietersnieuwstraat 41, 9000 Gent, Belgium \\ stijn.scheerlinck@intec.ugent.be
}

\begin{abstract}
Compact metal grating couplers for efficient and broadband coupling between optical fibers and silicon-on-insulator waveguides are proposed and demonstrated. Simulation results for silver, gold, aluminum and copper grating couplers are presented. Using a uniform silver grating, coupling efficiencies are calculated as high as $60 \%$ at a wavelength of 1.55 $\mu \mathrm{m}$. Metal grating couplers require only one single etching or lift-off step and are therefore very easy to fabricate. As a proof of principle, a gold grating coupler for near-vertical fiber-to-waveguide coupling was fabricated using e-beam writing and lift-off, demonstrating $34 \%$ coupling efficiency and a $1 \mathrm{~dB}-$ bandwidth of $40 \mathrm{~nm}$. Measurement data and simulation results are in good agreement.
\end{abstract}

(C) 2007 Optical Society of America

OCIS codes (130.0130) Integrated optics; (050.2770) Gratings.

\section{References and links}

1. W. Bogaerts, R. Baets, P. Dumon, V. Wiaux, S. Beckx, D. Taillaert, B. Luyssaert, J. Van Campenhout, P. Bienstman, and D. Van Thourhout, "Nanophotonic waveguides in silicon-on-insulator fabricated with CMOS technology," J. Lightwave Technol. 23, 401-412 (2005).

2. D. Taillaert, P. Bienstman, and R. Baets, "Compact efficient broadband grating coupler for silicon-oninsulator waveguides," Opt. Lett. 29, 2749--2751 (2004).

3. D. Taillaert, R. Baets, P. Dumon, W. Bogaerts, D. Van Thourhout, B. Luyssaert, V. Wiaux, S. Beckx, J. Wouters, "Silicon-on-Insulator platform for integrated wavelength-selective Components," Proc. of IEEE/LEOS Workshop on Fibers and Optical Passive Components, 115--120 (2005).

4. F. Van Laere, G. Roelkens, M. Ayre, J. Schrauwen, D. Taillaert, D. Van Thourhout, T. F. Krauss, and R. Baets, "Compact and highly efficient grating couplers between optical fiber and nanophotonic waveguides," J. Lightwave Technol. 25, 151--156 (2007).

5. G. Roelkens, D. Van Thourhout, and R. Baets, "High efficiency Silicon-on-Insulator grating coupler based on a poly-Silicon overlay," Opt. Express, 14, 11622--11630 (2006).

6. P. Bienstman and R. Baets, "Optical modeling of photonic crystals and VCSEL's using eigenmode expansion and perfectly matched layers," Opt. Quantum Electron. 33, 349-354 (2001).

7. P. B. Johnson and R. W. Christy, "Optical Constants of the Noble Metals," Phys. Rev. B 6,4370-4379 (1972).

8. E. D. Palik, "Handbook of Optical Constants of Solids," Academic Press Inc. (1985), ISBN 0-12-544420-6.

9. R. Emmons and D. Hall, "Buried-oxide silicon-on-insulator structures II: Waveguide grating couplers," J. Quantum Electron. 28, 164-175 (1992).

10. T. Suhara and H. Nishihara, "Integrated optics components and devices using periodic structures," J. Quantum Electron. 22, 845-867 (2002).

11. K. A. Bates, L. Li, R. Roncone, and J. Burke, "Gaussian beams from variable groove depth grating couplers in planar waveguides," Appl. Opt. 32, 2112-2116 (1993).

12. R. Waldhausl, B. Schnabel, P. Dannberg, E. Kley, A. Brauer, W. Karthe, "Efficient coupling into polymer waveguides by gratings," Appl. Opt. 36, 9383-9390 (1997).

\section{Introduction}

Silicon-on-Insulator (SOI) is emerging as a very interesting platform for integrated nanophotonics due to the high refractive index contrast between the silicon core and the oxide cladding $(\Delta \mathrm{n} \approx 2)$. This material system is very well suited for high density integration of photonic components and circuits which can be fabricated by standard CMOS technology [1]. 
One of the drawbacks of the high index contrast is the large mismatch in mode size and shape between the fundamental mode of SOI waveguides and the mode of optical fibers. This makes efficient coupling from fiber to waveguide difficult to achieve. Grating couplers defined by etching a grating in the high index silicon waveguide layer offer a very elegant solution to this problem [2]. Their compactness $( \pm 10 \mu \mathrm{m} \times 10 \mu \mathrm{m})$, efficiency, large optical bandwidth and very good alignment tolerances make them suitable for applications such as rapid testing of photonic circuits and packaging with optical in- and outputs.

For SOI grating couplers, a coupling efficiency of $33 \%$ has been demonstrated [3], and several approaches are being investigated to improve the coupling performance. In particular, introducing a bottom gold mirror improves the coupling efficiency up to $69 \%$ [4] and apodizing the grating in combination with local deposition of a poly-silicon overlay is expected to improve this even more [5]. However, all of these approaches require a number of extra processing steps and make fabrication rather challenging. In this paper, we propose and demonstrate a novel grating coupler device that is very easy to fabricate and which offers a very good alternative to conventional SOI grating coupler devices in terms of coupling efficiency and optical bandwidth.

\section{Device lay-out}

The metal grating coupler layout is depicted in Fig. 1(a). It consists of a metal grating on top of the silicon waveguide layer of an SOI layer structure. The metals we consider in this paper are gold, silver, copper and aluminum. The optical fiber is tilted with respect to the vertical axis as the vertical position leads to a large second order reflection. An index matching glue can be introduced between the optical fiber and the grating coupler so that reflections at the fiber facet are avoided. At a wavelength of $1.55 \mu \mathrm{m}$, there is a very high refractive index contrast between the metal teeth and their surroundings (the refractive index contrast between silver and silicon for example reads $\Delta \mathrm{n}=\mathrm{n}_{\mathrm{Si}}-\mathrm{n}_{\mathrm{Ag}} \approx 3.3+11.3 j$ ). Thus, light incident on the grating is strongly scattered. This property is exploited here for the design of efficient, broadband and compact grating couplers. Although a metal and thus lossy material is introduced, very high efficiencies are obtained due to the strong scattering on a small length scale. Figure 1(b) shows an example of a fabricated $10 \mu \mathrm{m}$ x $10 \mu \mathrm{m}$ gold grating coupler on top of an SOI waveguide.

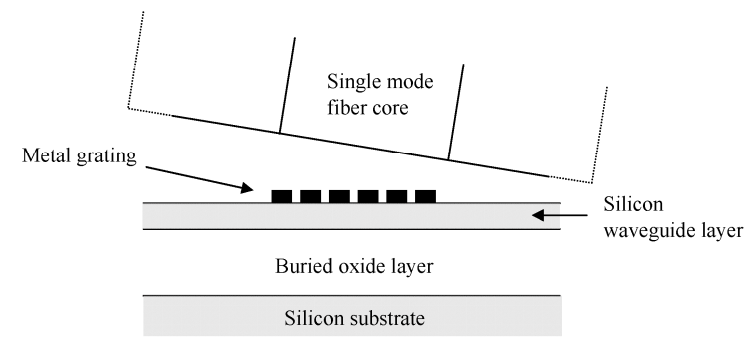

(a)

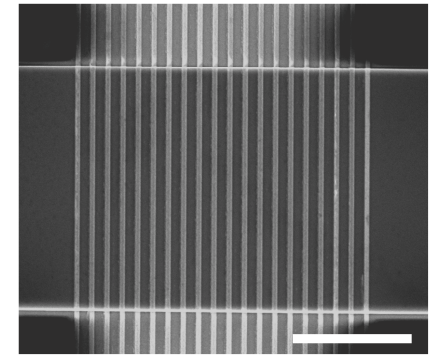

(b)

Fig. 1. Metal grating on top of an SOI-waveguide for coupling to a single mode optical fiber. (a) Device layout (side view), (b) SEM-image of a fabricated device (top view, bar length $=5$ $\mu \mathrm{m})$.

\section{Design and simulation}

For the design of metal grating couplers and optimization, we use CAMFR, a twodimensional fully vectorial simulation-tool based on eigenmode expansion and mode propagation with perfectly matched layer (PML) boundary conditions [6]. We consider 1-D gratings in 2-D simulations. Although the coupling problem is a 3D-problem, a 2-D approximation is valid for waveguides that are wide enough [2]. The proposed grating coupler 
layout can be used for coupling TE- (electric field parallel to the grating lines) as well as TMpolarized light (magnetic field parallel to the grating lines). Due to the difference in effective index between the fundamental TE- and TM-mode of SOI waveguides, the grating period of a TE grating coupler is different from the grating period of a TM grating coupler. In this paper, we concentrate on TE polarization. Refractive index data for gold, silver and copper were taken from [7] and for aluminum from [8]. The waveguide mode is incident from the left and is normalized to the input power. The refractive index of the area between grating and fiber is matched to the index of the fiber, representing an index matching glue. The top and bottom boundaries of the simulation area have PML to avoid parasitic reflections. In a first step reflection and transmission of the entire structure is calculated. Second, the power flux and overlap with the fiber mode is calculated from the field diffracted by the grating. The fraction of the input power that couples to the fiber mode is defined as the coupling efficiency. To this end, the fiber mode is modeled as a Gaussian distribution with a full width at half maximum (FWHM) of $5.2 \mu \mathrm{m}$.

Uniform metal grating couplers were studied on an SOI layer stack with top silicon layer thickness of $220 \mathrm{~nm}$. These grating couplers are characterized by the grating period, height and filling factor, whereby the filling factor is defined as the metal fraction of the grating period. Coupling is aimed for between the first diffraction order of the grating and the fundamental TE mode supported by a $10 \mu \mathrm{m}$ wide and $220 \mathrm{~nm}$ high SOI waveguide. From Bragg's condition a period of $600 \mathrm{~nm}$ is found when the first order diffraction is 10 degrees off the vertical direction. Using CAMFR, the grating period and other grating parameters are further optimized so that the grating directionality, defined as the fraction of the input power in the waveguide that is diffracted in the upward direction, is maximal. In Fig. 2, the result is plotted for silver gratings for an optimal value of the grating period. It follows from this simulation that even with a silver layer as thin as $5 \mathrm{~nm}, 50 \%$ of the incoming light is diffracted upward. Moreover, the grating directionality can be optimized considerably by a good choice of filling factor and grating height. The simulation results also indicate that the design is tolerant for errors in these parameters as the grating directionality varies only slightly for optimal parameters.

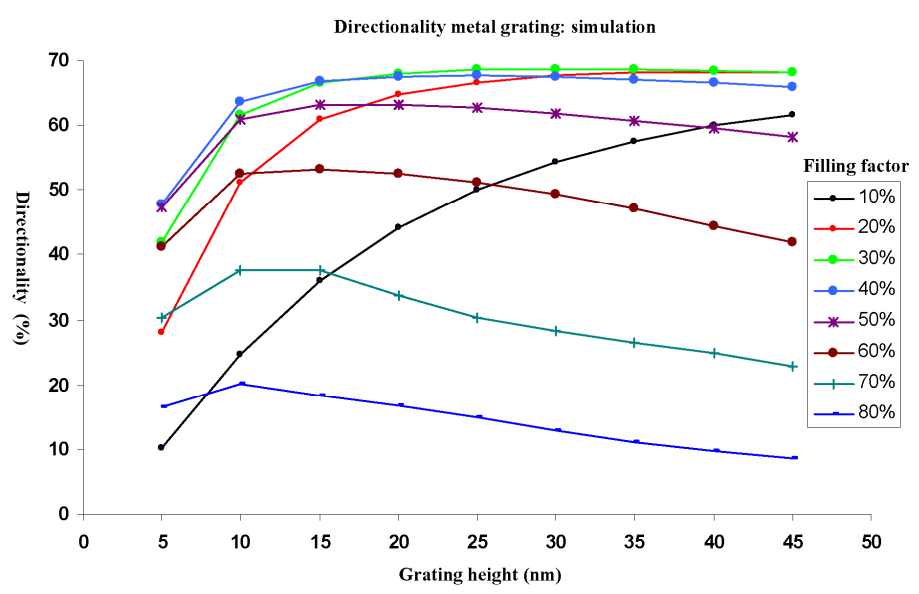

Fig. 2. Simulation of metal grating directionality as a function of grating height. The metal is silver. Buried oxide layer thickness is $1.35 \mu \mathrm{m}$. Period of the grating is $610 \mathrm{~nm}$.

For an optimal set of grating parameters (ie. period $610 \mathrm{~nm}$, filling factor $30 \%$, grating height $20 \mathrm{~nm}$ ) and a given buried oxide layer thickness of $1.35 \mu \mathrm{m}$, simulation shows that the direction of the outcoupled field at a wavelength of $1.55 \mu \mathrm{m}$ is tilted 9 degrees with respect to the vertical, as is illustrated by the field plot in Fig. 3. For a fixed fiber tilt, the coupling efficiency as a function of wavelength is plotted in Fig. 4. For comparison, the coupling 
efficiency of a silver, gold, copper and aluminum grating for the same grating parameters are plotted in the same graph. Although the optimal grating parameters differ for the different metals considered, these simulation results indicate that highly efficient and broadband coupling can be obtained, also with CMOS-compatible metals such as copper and aluminum. Coupling efficiencies as high as $60 \%, 54 \%, 52 \%$ and $50 \%$ are calculated for silver, gold, copper and aluminum gratings respectively. For all gratings, the $1 \mathrm{~dB}$ bandwidth is about 40 $\mathrm{nm}$.

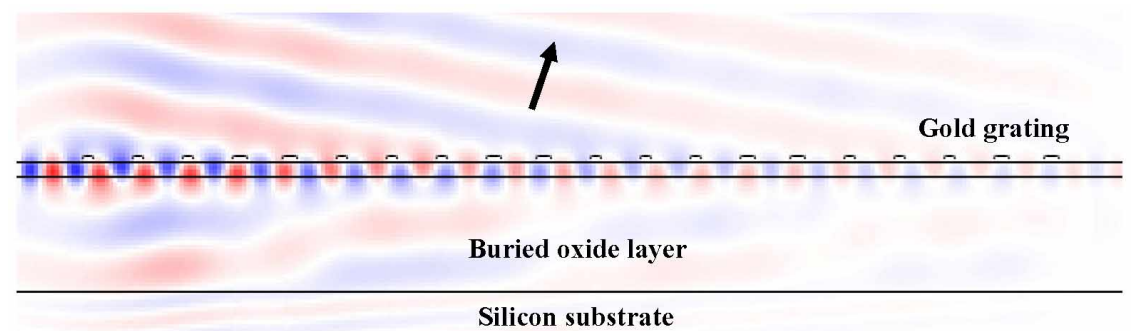

Fig. 3. Field plot for an optimized uniform silver grating. Grating period $=610 \mathrm{~nm}$, filling factor $=30 \%$, thickness of the silver layer $=20 \mathrm{~nm}$, buried oxide layer thickness $=1.35 \mu \mathrm{m}$.

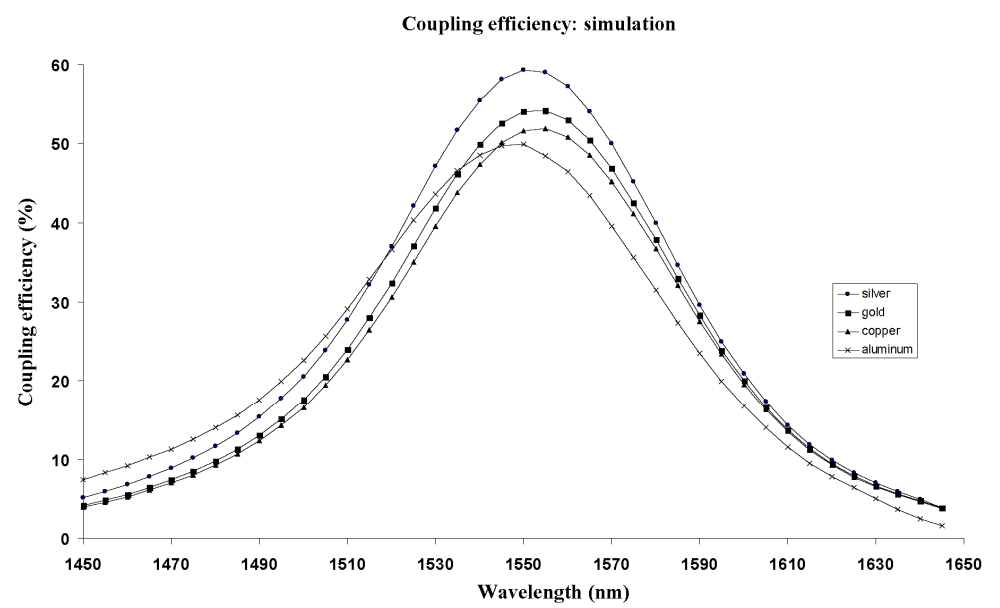

Fig. 4. Simulation of the coupling efficiency for silver, gold, aluminum and copper grating couplers for SOI waveguides. Grating period $=610 \mathrm{~nm}$, filling factor $=30 \%$, grating height $=$ $20 \mathrm{~nm}$, buried oxide layer thickness $=1.35 \mu \mathrm{m}$, fiber tilt $=9$ degrees.

When designing grating couplers for near-vertical coupling, the buried oxide layer thickness is a key-parameter as it is of great influence on the coupling efficiency. In Fig. 5, the coupling efficiency of a silver grating coupler with period $610 \mathrm{~nm}$, filling factor $30 \%$ and grating height $20 \mathrm{~nm}$ to a fiber tilted 9 degrees with respect to the vertical is plotted as a function of buried oxide layer thickness. The periodic behavior is a well-known property of grating couplers [9], [10]. This effect can be explained as follows: the grating coupler creates an upward propagating and a downward propagating wave. The downward wave partially reflects at the oxide-substrate interface and interferes with the direct upward wave. Consequently, only for optimal values of the buried oxide layer thickness, constructive interference occurs, resulting in maxima in the grating directionality and thus in coupling efficiency. Depending on the buried oxide layer thickness, the coupling efficiency varies between $20 \%$ and $60 \%$. 


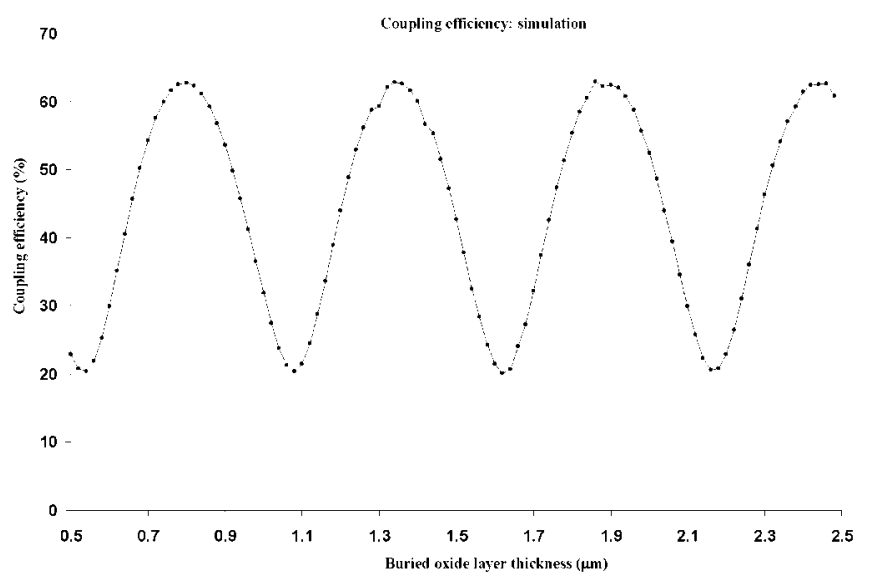

Fig. 5. Simulation of the coupling efficiency of a silver grating coupler as a function of buried oxide layer thickness.

The results presented in this work are valid for uniform metal gratings. We note that the coupling efficiency between SOI waveguides and single-mode fibers can be further improved by optimizing the individual teeth and slit widths of the metal grating so as to reduce the mismatch between the exponentially decaying power profile of the light diffracted by the grating and the Gaussian profile of the optical fiber [2], [11], [12].

\section{Fabrication}

The ease of fabrication is a key property of metal grating couplers. Processing schemes based on lift-off or etching are possible. As a proof of principle, we fabricated gold grating couplers for near-vertical coupling on top of SOI waveguides with e-beam lithography followed by lift-off. The SOI layer structure of the sample used consisted of a buried oxide layer of thickness $2 \mu \mathrm{m}$ and top silicon layer of thickness $220 \mathrm{~nm}$.

The grating coupler fabrication scheme was the following. First, host waveguides of width $10 \mu \mathrm{m}$ and height $220 \mathrm{~nm}$ were fabricated using Deep UV-lithography (DUV) and inductively coupled plasma etching (ICP). Then, a $400 \mathrm{~nm}$ PMMA e-beam resist with $950 \mathrm{k}$ molecular weight (Brewer Science, Inc.) was spun and baked for 2 minutes at $180{ }^{\circ} \mathrm{C}$ before e-beam writing. We used the electron beam of a Nova 600 Dual-beam (FEI) system for the grating definition on top of the waveguide. The sample was then developed in an MIBK:IPA (1:3) solution. Prior to gold evaporation, $3 \mathrm{~nm}$ of titanium was sputtered to enhance adhesion between the gold and the silicon. Finally, lift-off was performed in acetone. In this way two grating couplers were fabricated on the same waveguide. The grating parameters were measured after fabrication and found to be the following: period $630 \mathrm{~nm}(20 \mathrm{~nm}$ off target) and filling factor $30 \%$ (on target). A SEM-picture of the fabricated device is depicted in Fig. $1(\mathrm{~b})$.

\section{Measurement results}

The gold grating couplers were optically characterized using the following experiment. A single mode fiber connected to a tunable laser source was positioned over the first grating coupler and another fiber connected to a photodetector was positioned over the second grating coupler in the presence of index matching glue. Polarization wheels were used to control polarization. After an active alignment procedure whereby the fiber positions were optimized in order to obtain maximal transmission, the wavelength of the light was swept. From this measurement, the fiber-to-waveguide coupling efficiency of a single gold grating coupler is calculated and plotted in Fig. 6. A fiber-to-waveguide coupling efficiency of $34 \%$ and a $1 \mathrm{~dB}$ bandwidth of $40 \mathrm{~nm}$ has been measured. The result of the CAMFR-simulation of the structure 
was added for comparison. The discrepancy between the simulation and experimental result in terms of efficiency is caused by the presence of the absorbing $\mathrm{Ti}$ adhesion layer, grating defects and roughness and the fact that the grating lines are not perfectly perpendicular to the waveguide axis.

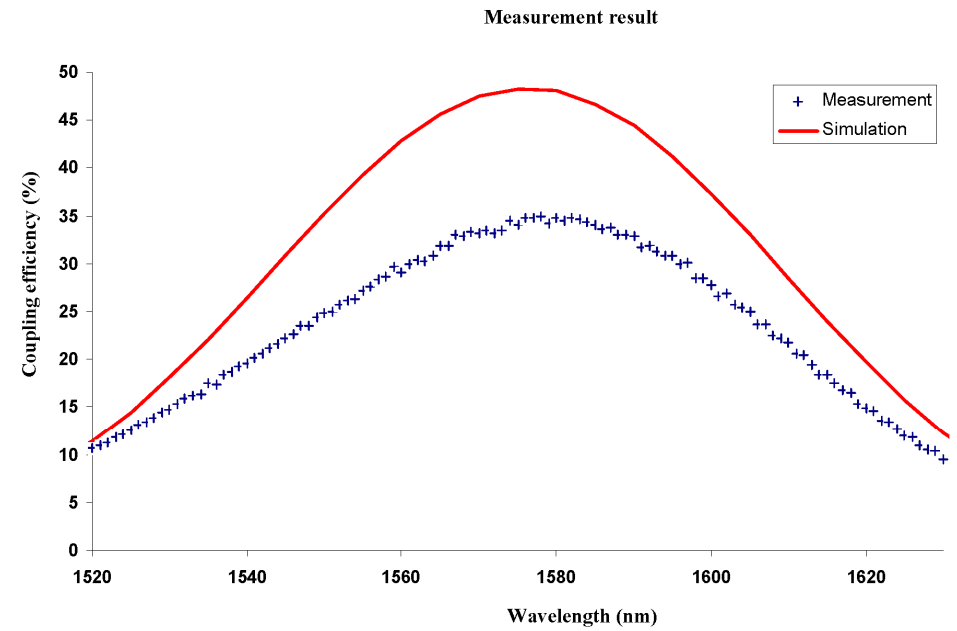

Fig. 6. Experimentally determined fiber-to-waveguide coupling efficiency of a gold grating coupler with parameters: period $=630 \mathrm{~nm}$, filling factor $=30 \%$, grating height $=20 \mathrm{~nm}$ with a $3 \mathrm{~nm}$ Ti adhesion on top of an SOI waveguide using a single-mode fiber tilted 10 degrees with respect to the vertical. CAMFR-simulation results are added for comparison.

\section{Conclusion}

In this paper, we proposed metal grating couplers for efficient and broadband coupling between SOI waveguides and optical fibers. We studied the performance of these grating couplers by simulation and designed metal gratings with coupling efficiencies exceeding $50 \%$ at a wavelength of $1550 \mathrm{~nm}$. Metal grating couplers are easy to fabricate using a single etching or lift-off step. A gold grating coupler prototype has been fabricated for coupling to SOI waveguides using e-beam lithography and lift-off demonstrating $34 \%$ coupling efficiency at a wavelength of $1580 \mathrm{~nm}$ and a $1 \mathrm{~dB}$ bandwidth of $40 \mathrm{~nm}$. The experimental results are in good agreement with simulation results.

\section{Acknowledgments}

The authors would like to acknowledge Ghent University for a GOA grant, the Belgian IAPPHOTON Network and the European Union for its support through the IST-ePIXnet Network of Excellence. Stijn Scheerlinck and Frederik Van Laere thank the Institute for the Promotion of Innovation through Science and Technology in Flanders (IWT-Vlaanderen) for a specialization grant. Dirk Taillaert thanks the Institute for the Promotion of Innovation through Science and Technology in Flanders (IWT-Vlaanderen) for a post-doctoral grant. 